\title{
Do response time advantage and interference reflect the order of processing of global- and local-level information?
}

\author{
MARVIN R. LAMB and LYNN C. ROBERTSON \\ Veterans Administration Medical Center, Martinez, California, \\ and School of Medicine, University of California, Davis, California
}

\begin{abstract}
Navon's (1977) global precedence hypothesis was based primarily on the joint occurrence of two effects: a response time (RT) advantage for identifying global targets, and interference by global distractors on responding to local targets. Although the hypothesis has been questioned on the basis of experiments in which it has been shown that a local RT advantage and local interference can occur, it is still frequently assumed that these two effects are a valid measure of the order in which local and global levels of structure are processed. In the present experiment, this assumption was examined. Subjects identified target letters that occurred randomly at the global or local level in a divided-attention task. The visual angle subtended by the stimulus pattern was varied, a manipulation known to affect the relative speed of response to local- or global-level information. Local targets were identified faster than global targets at the larger visual angles, but there was no difference in RT at the smallest visual angle. Despite this change in RT advantage, the interference effect did not change as a function of the visual angle of the stimulus pattern. Moreover, global distractors interfered with responding to local targets but local targets had no effect on responding to global targets, which is exactly the opposite of the finding one would expect if RT advantage and interference reflected order of processing. These findings are not consistent with the assumption that RT advantage and interference reflect order of processing in a simple way.
\end{abstract}

The global precedence hypothesis (Navon 1977, 1981) asserts that global-level information is processed before local-level information and has been based primarily on the joint occurrence of two effects: a global advantage in response time (RT), and global interference. In a number of studies, it has been found that subjects identify global targets (e.g., the large letters in Figure 1) more quickly than they identify local targets (e.g., the small letters in Figure 1). At the same time, global distractors slow responses to local targets, but local distractors have little or no effect on responses to global targets. The fact that global information interferes with the local response but not vice versa has been taken as evidence that information at the global level is present at the time of the local response but not the reverse.

Although these two effects (i.e., a global advantage in $\mathrm{RT}$, and global interference) have frequently been reported (Boer \& Keuss, 1982; Grice, Canham, \& Boroughs, 1983; Lamb \& Robertson, 1988; Martin, 1979a; Navon, 1977; Navon \& Norman, 1983; Pomerantz, 1983), they are by

This research was supported by the Medical Research Council of the Veterans Administration and NIAAA Grant AA06637 to the second author. We wish to thank Linda Kerth for her help with data collection and figure preparation, and John Lackey for computer programming. Correspondence concerning this article should be sent to Marvin $\mathbf{R}$. Lamb, Research 151, Veterans Administration Medical Center, 150 Muir Road, Martinez, CA 94553. no means universal. Changes in the relative discriminability of local and global forms, the sparsity of local forms, the size or brightness of the stimulus pattern, and where on the retina the stimulus impinges have all been shown to produce a reduction or reversal in the global advantage in RT accompanied by a reduction or reversal of global interference (Boer \& Keuss, 1982; Grice et al., 1983; Kinchla \& Wolfe, 1979; Martin, 1979b; Pomerantz, 1983). For example, Martin (1979b) reduced the number of local elements in patterns similar to those in Figure 1 and found a local advantage in RT and also that local distractors interfered with global responses but not vice versa.

Such data create problems for the global precedence hypothesis, because they suggest that, at least under some conditions, local processing precedes global processing. However, this conclusion depends on the assumption that the level of the RT advantage together with the level of interference reflects order of processing-an assumption brought into question by several recent findings. If RT advantage and interference reflect order of processing, then they should covary in a systematic way. Global precedence should be reflected in global advantage, and global interference and local precedence should be reflected in local advantage and local interference. However, several studies have shown that RT advantage and interference do not always vary in the expected manner. For example, Navon and Norman (1983) reported a situation in which there was a large global advantage in RT, but local 


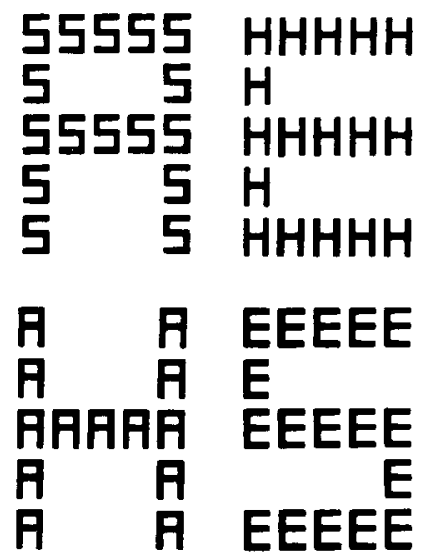

Figure 1. Drawings of four (of the eight) stimuli used in the present experiment.

distractors slowed responding to global targets just as much as global distractors slowed responding to local targets. Lamb and Robertson (1988) found that large changes in RT advantage can occur without a corresponding change in interference. Perhaps most compelling of all are data from brain-injured patients, showing that RT advantage and interference can be independently impaired (Humphreys, Riddoch, \& Quinlan, 1986; Lamb, Robertson, \& Knight, 1989). For example, Lamb et al. found that patients with lesions centered in temporal regions showed no interference effects, whereas patients with lesions centered in parietal regions showed normal interference. This was the case even though both groups showed the normal global advantage in RT. These findings suggest that RT advantage and interference can be determined by separate mechanisms and do not simply reflect the order in which the different levels of a stimulus are processed.

The present study was designed to further explore the relation between $\mathrm{RT}$ advantage and interference. In all the studies just described, a selective-attention paradigm was employed. That is, the subjects were informed in advance which level was relevant, so that attention could be directed to that level. The present study involved a divided-attention paradigm in which the subjects identified target letters that appeared randomly at either the local or the global level. Since the subjects did not know in advance which level would contain the target, both levels had to be monitored. RT advantage can be measured in the same way for both selective- and divided-attention paradigms as simply the difference in performance in identifying local versus global targets. However, interference cannot be measured in the divided-attention paradigm in the same way as it has in the selective-attention paradigm. In the selective-attention paradigm, interference has been measured as the difference in performance when the letters at the two levels are associated with the same response (e.g., global " $H$ ", local " $H$ ") as compared with when they are associated with different responses (e.g., global
"H", local " $\mathrm{S}$ "). Since subjects are told in advance to respond only to letters at one level or the other, the task is unambiguous even when the two levels contain letters associated with different responses. This is not the case in the divided-attention task, however. Since both levels are always relevant, the task would be impossible if the two levels contained letters associated with incompatible responses. However, it has been shown that systematic interference effects can be obtained through varying the similarity relations between target and distractor stimuli even if responses are associated only with target stimuli (B. A. Eriksen \& C. W. Eriksen, 1974). We took advantage of this fact in the present experiment. The letters " $H$ " and " $S$ " were chosen as targets, and the letters " $A$ " and " $E$ "' as distractors. As can be seen in Figure 1, the letters $\mathrm{A}$ and $\mathrm{H}$ were identical, apart from the addition of the top horizontal segment in the $A$, and the letters $E$ and $S$ were identical, except that the lower vertical segment was on the left for the $E$ and on the right for the $S$. Thus, $A$ was more similar to $\mathrm{H}$ than to $\mathrm{S}$, and $\mathrm{E}$ was more similar to $S$ than to $\mathrm{H}^{1}{ }^{1}$ Given these similarity relations, RTs to $S$ targets should be faster with $E$ than with $A$ as the distractor, while RTs to $\mathrm{H}$ targets should be faster with $A$ than with $E$ as the distractor. In other words, interference can be measured as the interaction between target and distractor letters.

Finally, in order to see whether changes in RT advantage would be accompanied by changes in interference in a divided-attention task, we also varied the visual angle of the stimulus patterns, a manipulation known to affect relative RTs to local and global letters (Kinchla \& Wolfe, 1979).

\section{METHOD}

\section{Subjects}

Ten right-handed, paid volunteers served as subjects. They ranged in age from 48 to 70 years $(M=60.7, S D=7.3)$. All subjects had normal or corrected-to-normal vision.

\section{Apparatus and Stimuli}

The stimuli were generated on a Princeton Graphics SR-12 monitor controlled by an IBM XT computer with Sigma Designs Graphic Dazzler I and Enhancer cards. All stimulus events were white on a dark surround. Stimulus timing (onset, offset, and duration) was tied to the vertical sync pulse. All other events (responses, ITI, etc.) were timed with the 8253 chip set to a 1 -msec time base. The status of the response keys was monitored via the game port.

A set of stimulus patterns formed from the letters $\mathrm{H}, \mathrm{S}, \mathrm{A}$, and $E$ was used (see Figure 1). Global letters were constructed from the appropriate placement of local letters in a $5 \times 5$ matrix. In separate blocks of trials, the global pattern subtended $3^{\circ}, 6^{\circ}, 9^{\circ}$, or $12^{\circ}$, vertically. Global letters were 7.4 times as tall as local letters. Both global and local letters were 1.5 times as tall as they were wide.

The letters $\mathrm{H}$ and $\mathrm{S}$ served as targets, because these letters have frequently been used as targets in studies supporting the global precedence hypothesis. The letters $A$ and $E$ served as distractors. All possible combinations of these letters appeared equally often, with each stimulus containing one target and one distractor letter. Thus there were eight stimuli in all: global $\mathrm{H}$ and local $\mathrm{A}$, global $\mathrm{H}$ and local $E$, global $S$ and local $A$, global $S$ and local $E$, local $H$ and 
global A, local H and global E, local S and global A, local S and global E. Four of these are shown in Figure 1. All of the stimuli appeared at the center of the screen.

\section{Procedure}

The subjects sat with their heads resting against the back of a large easy chair. The distance between their eyes and the CRT screen was approximately $71 \mathrm{~cm}$. A .5-sec tone announced the beginning of each trial. The tone was followed by a .1-sec pause and then by a .1-sec presentation of one of the stimulus patterns. The subject's task was to indicate, by pressing one of two response keys, which of the target letters $(H$ or $S$ ) was present on that trial. A 1-sec ITI followed the response, or the next trial was presented if no response occurred within $4 \mathrm{sec}$ of stimulus presentation.

Each subject received 640 trials in all, of which 128 were practice. There were five blocks of trials on each of 2 days of testing. On the first day, the subjects received a block of 48 practice trials with $12^{\circ}$ stimuli, followed by a block of 68 trials at each of the four visual angles in descending order. On the second day, they received a block of 48 practice trials with $3^{\circ}$ stimuli, followed by a block of 68 trials at each of the four visual angles in ascending order. The 48-trial blocks along with the first four trials of cach 68-trial block were considered practice and were not included in the analyses.

The stimuli were presented randomly, with the restriction that target letter ( $\mathrm{H}$ and $\mathrm{S}$ ), distractor letter (A and $\mathrm{E}$ ), and target level (local and global) be counterbalanced within each block.

The subjects used their right hands to respond and pressed the $\mathrm{H}$ key with their index fingers and the $\mathrm{S}$ key with their middle fingers. All subjects were instructed to respond as quickly as possible while keeping errors to a minimum.

\section{RESULTS AND DISCUSSION}

The data (both RTs and errors) were subjected to a repeated measures analyses of variance (ANOVA), with target level (local or global), visual angle $\left(3^{\circ}, 6^{\circ}, 9^{\circ}\right.$, or $\left.12^{\circ}\right)$, target letter ( $\mathrm{H}$ or $\left.\mathrm{S}\right)$, and distractor letter (A or $\left.\mathrm{E}\right)$ as factors. The error data are shown in Table 1 . The error rate was low $(M=1.3 \%)$, and the ANOVA revealed no significant effects. Median RT (correct trials only) was calculated for each cell in the design and the data reported in the figures and in the ANOVAs are means of those medians.

Table 1

Mean Percentage Errors

\begin{tabular}{|c|c|c|c|c|}
\hline \multirow{2}{*}{$\begin{array}{l}\text { Distractor } \\
\text { Letter }\end{array}$} & \multicolumn{4}{|c|}{ Target Letter } \\
\hline & Global H & Global S & Local $\mathbf{H}$ & Local S \\
\hline \multicolumn{5}{|c|}{$3^{\circ}$ Visual Angle } \\
\hline $\mathbf{A}$ & 0.0 & 0.6 & 0.0 & 3.8 \\
\hline $\mathbf{E}$ & 1.3 & 0.0 & 3.8 & 0.6 \\
\hline \multicolumn{5}{|c|}{$6^{\circ}$ Visual Angle } \\
\hline A & 0.0 & 0.0 & 0.0 & 4.4 \\
\hline $\mathbf{E}$ & 1.3 & 1.3 & 4.4 & 0.6 \\
\hline \multicolumn{5}{|c|}{$9^{\circ}$ Visual Angle } \\
\hline $\mathbf{A}$ & 0.6 & 0.6 & 0.6 & 2.5 \\
\hline $\mathrm{E}$ & 0.0 & 1.3 & 3.8 & 0.6 \\
\hline \multicolumn{5}{|c|}{$12^{\circ}$ Visual Angle } \\
\hline $\mathbf{A}$ & 0.6 & 0.6 & 0.6 & 5.0 \\
\hline $\mathbf{E}$ & 0.0 & 1.9 & 0.6 & 1.3 \\
\hline
\end{tabular}

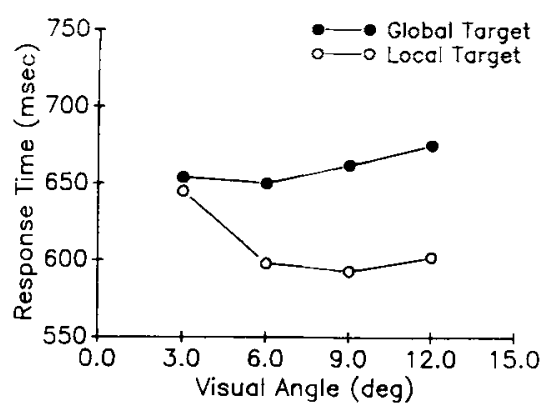

Figure 2. Response time as a function of the visual angle of the stimulus pattern for global targets (closed circles) and local targets (open circles).

Consistent with earlier findings (Kinchla \& Wolfe, 1979), the relative speed of responding to local and global targets varied as a function of the visual angle of the stimulus pattern (see Figure 2). Although neither the main effect of target level nor visual angle reached statistical significance $[F(1,9)=4.51, p<.06$, and $F(3,27)=2.60$, $p<.07$, respectively], the target level $\times$ visual angle interaction was significant $[F(3,27)=4.87, p<.01]$. This interaction reflects the fact that local RTs were faster than global RTs for the three largest patterns $[F(1,9)=$ $6.05, p<.05]$, whereas global and local RTs did not differ when the pattern subtended only $3^{\circ}(F<1)$.

These data differ from those of Kinchla and Wolfe (1979) in that a global advantage was never found-even at the smallest visual angle used. Kinchla and Wolfe found a local advantage at visual angles greater than about $6^{\circ}$ and a global advantage at smaller visual angles. One possible reason for this discrepancy might be that the present subjects were considerably older than those in the Kinchla and Wolfe study. Age differences can have a dramatic effect on performance in RT tasks (Krueger \& Allen, 1987). However, recent findings from our laboratory suggest that age is not the crucial factor in this case (Lamb \& Robertson, 1989). Using the same older subjects who participated in the present experiment, we found that the visual angle at which the transition from a global to a local advantage in RT occurred did not differ for older (mean age $=60.7$ ) and younger (mean age $=23.2$ ) groups. On the other hand, the visual angle at which the transition occurred did depend on the set of visual angles to which subjects had been exposed (regardless of age). Thus it is possible that the discrepancy between the present results and those of Kinchla and Wolfe (1979) reflects the fact that the set of visual angles differed in the two studies. In any event, the important finding for the present purpose is that variation in visual angle produced a variation in RT advantage.

As we had found previously (Lamb \& Robertson, 1988), there were large and systematic differences in RT, depending on the particular letters in the display. Although neither the main effect of target letter nor that of distractor letter reached statistical significance $(F \mathrm{~s}<1)$, the target letter $\times$ distractor letter interaction was significant 
$[F(1,9)=16.26, p<.01]$. This interaction reflected the fact that RTs were faster for $H$ than for $S$ targets if the distractor was an $\mathrm{A}$, but faster for $\mathrm{S}$ than for $\mathrm{H}$ targets if the distractor was an $\mathrm{E}$ (see Figure 3). This pattern of interference effects is similar to earlier findings with a nonglobal/local task (B. A. Eriksen \& C. W. Eriksen, 1974), and it is consistent with the response-competition model proposed by Eriksen and his associates (C. W. Eriksen, O'Hara, \& B. [A.] Eriksen, 1982; C. W. Eriksen \& Schultz, 1979). Thus, as expected, distractor letters interfered with the processing of target letters. However, as is shown in Figure 3, this was the case for local but not
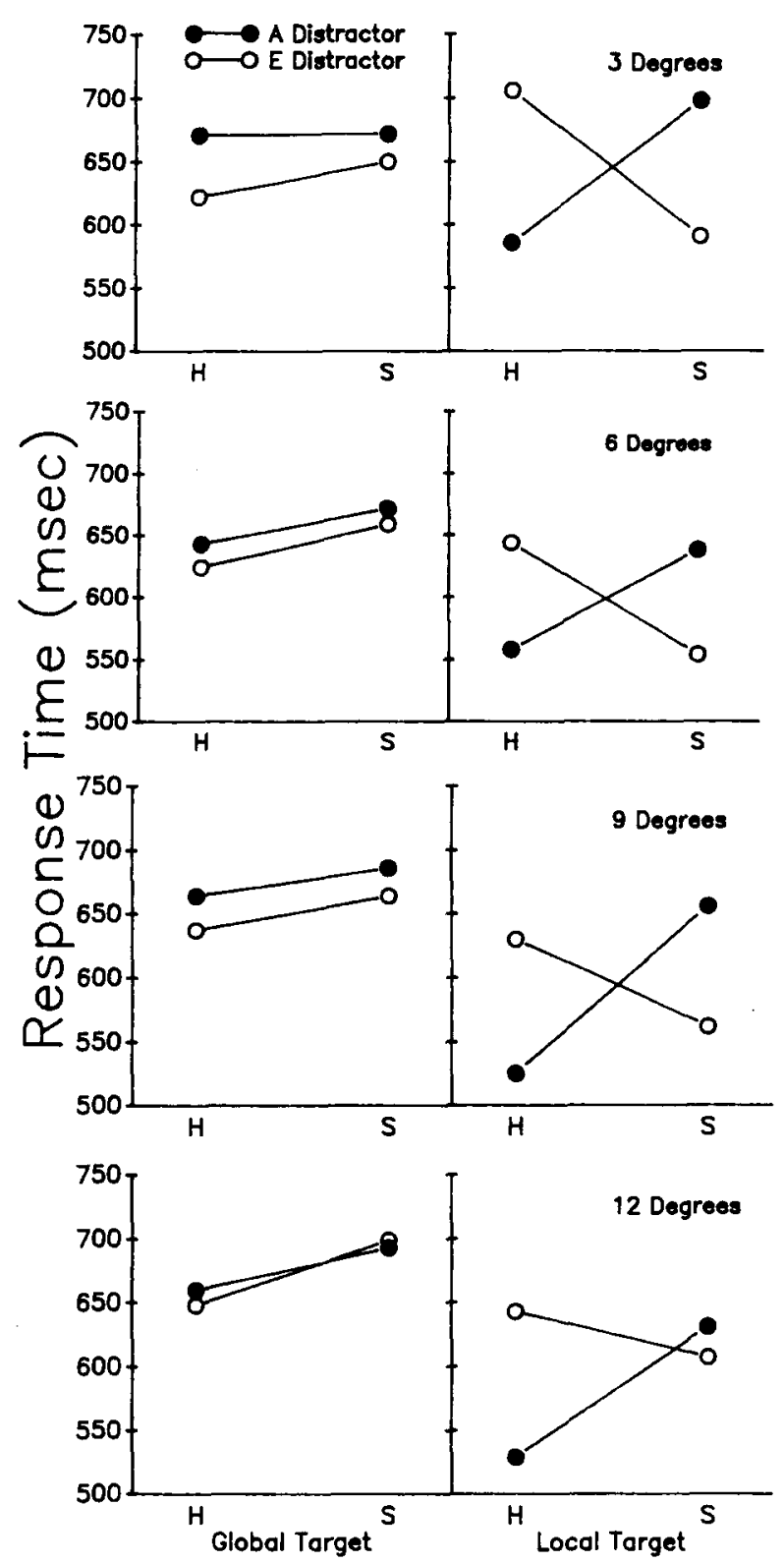

Figure 3. Response times for $\mathrm{H}$ and $\mathrm{S}$ targets when the distractor letter was an A (closed circles) or an $\mathbf{E}$ (open circles) for global targets (left panels) and local targets (right panels). Data are presented separately for each of the four visual angles. for global targets-a difference reflected in a significant target level $\times$ target letter $\times$ distractor letter interaction $[F(1,9)=27.44, p<.001]$. Thus, as with more traditional measures of interference (Grice et al., 1983; Lamb \& Robertson, 1988; Martin, 1979a; Navon, 1977; Navon \& Norman, 1983; Pomerantz, 1983), the present data show a marked asymmetry in the degree to which letters at one level influence the processing of letters at the other level.

It has frequently been asserted that such an asymmetry, combined with a level advantage in RT, reflects the order of processing of local and global levels. However, the present data clearly do not support this conclusion. First there was, if anything, a local advantage in RT, and so one would have expected local distractors to interfere with global processing more than the reverse. Instead, the exact opposite result was obtained. Distractors at the global level interfered more with local responses than distractors at the local level interfered with global responses. Furthermore, as already reported, the local advantage in RT declined as a function of the visual angle of the pattern. However, despite this change in RT advantage, visual angle had little or no effect on the asymmetrical influence of local versus global distractors $(F<1)$. As Figure 3 shows, the crossover target letter $\times$ distractor letter interaction occurred for local but not global targets at each visual angle, even though the local advantage in RT decreased dramatically from $12^{\circ}$ to $3^{\circ}$. Inspection of Figure 3 suggests that there might be some evidence of a decreased interference effect in the $12^{\circ}$ condition. However, a comparison of the $3^{\circ}$ and $12^{\circ}$ conditions produced no statistical difference in interference despite the presence of a large difference in RT $[F(1,9)=10.65$, $p<.01]$. It could also be argued that there is some indication of a main effect of distractor for global targets, and that this effect might vary as a function of visual angle. If so, it would be evidence that local distractors affected responding to global targets. However, the statistical analyses provided no support for this assertion. Neither the distractor letter $\times$ visual angle nor the distractor letter $X$ target letter $X$ visual angle interaction for global targets was significant $(F \mathrm{~s}<1)$.

The present data add to the growing body of evidence demonstrating that $\mathrm{RT}$ advantage and interference do not always bear the relationship one would expect if they were providing a simple measure of the order in which localand global-level information are processed. In fact, recent neuropsychological findings suggest that the orderof-processing question may be somewhat ill conceived, because there is good evidence that there are separate hemisphere-specific mechanisms capable of processing local- and global-level information (Delis, Robertson, \& Efron, 1986; Lamb et al., 1989; Robertson \& Delis, 1986; Robertson, Lamb, \& Knight, 1988). Thus it is not unlikely that local- and global-level information can be processed in parallel. In addition, it has recently been shown that unilateral temporal lobe lesions can eliminate interference effects without affecting RT advantage, 
strongly suggesting that interference does not simply reflect the order or even the relative speed with which global- and local-level information is processed (Lamb, Robertson, \& Knight, 1989). Of course data from braininjured patients are always subject to the argument that neural or cognitive reorganization has changed the way in which processes normally interact. However, the present data show that RT advantage and interference do not always covary even in subjects with an intact nervous system. Although the reasons behind this independence are not entirely clear, there is nevertheless a substantial body of converging evidence that separate mechanisms are responsible for $\mathrm{RT}$ advantage and interference, a fact that any theory of perceptual organization must take into consideration.

\section{REFERENCES}

Boer, L. C., Ke Keuss, P. J. G. (1982). Global precedence as a postperceptual effect: An analysis of speed-accuracy tradeoff functions. Perception \& Psychophysics, 31, 358-366.

Delis, D. C., Robertson, L. C., \& EFron, R. (1986). Hemispheric specialization of memory for visual hierarchical stimuli. Neuropsychologia, 24, 205-214.

ERIKSEN, B. A., \& ERIKSEN, C. W. (1974). Effects of noise letters upon the identification of a target letter in a nonsearch task. Perception \& Psychophysics, 16, 143-149.

Eriksen, C. W., O'Hara, W. P., \& Eriksen, B. [A.] (1982). Response competition effects in same-different judgments. Perception \& Psychophysics, 32, 261-270.

ERIKSEN, C. W., \& Schultz, D. W. (1979). Information processing in visual search: A continuous flow conception and experimental results. Perception \& Psychophysics, 25, 249-263.

Grice, G. C., Canham, L., \& Boroughs, J. M. (1983). Forest before trees? It depends where you look. Perception \& Psychophysics, 33, 121-128.

Humphreys, G. W., Riddoch, J., \& Quinlan, T. P. (1986). Interactive processes in perceptual organization: Evidence from visual agnosia. In M. I. Posner \& O. S. M. Marin (Eds.), Attention and performance $X I$ (pp. 301-318). Hillsdale, NJ: Erlbaum.

Kinchla, R. A., \& Wolfe, J. M. (1979). The order of visual processing: "Top-down," "bottom-up," or "middle-out." Perception \& Psychophysics, 25, 225-231.
Krueger, L. E., \& Allen, P. A. (1987). Same-different judgments of foveal and parafoveal letter pairs by older adults. Perception \& Psychophysics, 41, 329-334.

LAMB, M. R., \& RoberTson, L. C. (1988). The processing of hierarchical stimuli: Effects of retinal locus, locational uncertainty, and stimulus identity. Perception \& Psychophysics, 44, 172-181.

LAMB, M. R., \& ROBERTSON, L. C. (1989). The effect of visual angle on global and local reaction times depends on the set of visual angles presented. Manuscript submitted for publication.

LAmb, M. R., Robertson, L. C., \& KNIGHT, R. T. (1989). Attention and interference in the processing of hierarchical patterns: Inferences from patients with right and left temporal-parietal lesions. Neuropsychologia, 27, 471-483.

MarTIN, M. (1979a). Hemispheric specialization for local and global processing. Neuropsychologia, 17, 33-40.

MARTIN, M. (1979b). Local and global processing: The role of sparsity. Memory \& Cognition, 7, 476-484.

Navon, D. (1977). Forest before trees: The precedence of global features in visual perception. Cognitive Psychology, 9, 353-383.

Navon, D. (1981). Do attention and decision follow perception? Comment on Miller. Journal of Experimental Psychology: Human Perception \& Performance, 7, 1175-1182.

Navon, D., \&orman, J. (1983). Does global precedence really depend on visual angle? Journal of Experimental Psychology: Human Perception \& Performance, 9, 955-965.

Pomerantz, J. R. (1983). Global and local precedence: Selective attention in form and motion perception. Journal of Experimental Psychology: General, 112, 516-540.

Robertson, L. C., \& Delis, D. C. (1986). "Part-whole' processing in unilateral brain damaged patients: Dysfunction of hierarchical organization. Neuropsychologia, 24, 363-370.

Robertson, L. C., LAMB, M. R., KNiGHT, R. T. (1988). Effects of lesions of temporal-parietal junction on perceptual and attentional processing in humans. Journal of Neuroscience, 8, 3757-3769.

\section{NOTE}

1. We collected no independent measure of the similarity relation between target and distractor stimuli. However, none of the arguments made here depend on the similarity relations asserted. Our only goal was to find stimuli that would produce systematic interference effects, and this goal was achieved.

(Manuscript received September 6, 1988; revision accepted for publication February 28, 1989.) 